\title{
Effect of vibration on the stability of a gas-fluidized bed of fine powder
}

\author{
J. M. Valverde, A. Castellanos, and M. A. S. Quintanilla \\ Departamento de Electronica y Electromagnetismo, Universidad de Sevilla, Avenida Reina Mercedes s/n, 41012 Sevilla, Spain
}

(Received 18 January 2001; revised manuscript received 2 April 2001; published 18 July 2001)

\begin{abstract}
We have investigated the effect of vibrations on the stability of gas-fluidized beds of fine powders (particle size $\sim 10 \mu \mathrm{m}$ ). The powder is uniformly fluidized by an adjustable gas flow that enables us to control the average solid volume fraction $\phi_{0}$. The fluidized bed is then subjected to a vertical oscillatory motion of controlled amplitude and frequency. The response of the fluidized bed depends essentially on the value of $\phi_{0}$. For $\phi_{0}>0.28$ the fluidized bed is in a weak solidlike regime, it has a mechanical strength, and particles are static. In this regime vibration causes compaction of the loosely packed bed. For $\phi_{0}<0.28$ the mechanical strength vanishes and stresses are carried by interstitial gas and collisions. In this fluidlike regime the fluidized bed displays a diffusive dynamics and particles aggregate due to the strong interparticle van der Waals forces. When vibration is applied the powder expands due to the partial disruption of aggregates. However at a critical value of the vibration amplitude $A=A_{c}$ either surface (sloshing) or flow (bubbling) instabilities develop. The nucleation of gas bubbles has been correlated to the saturation in particle diffusivity measured elsewhere. The size of the bubbles increases as $A$ is further increased above $A_{c}$ and as the vibration frequency is reduced. Moreover, as it should be expected from the predictions of hydrodynamic models, $A_{c}$ is independent of cohesivity for particles of the same size and density.
\end{abstract}

DOI: 10.1103/PhysRevE.64.021302

PACS number(s): 45.70.-n, 47.55.Kf, 47.55.Mh

\section{INTRODUCTION}

Vibration of granular materials plays a fundamental role in industry, commonly used as a means of handling, transporting, mixing, or segregating these materials. Beds of noncohesive grains (typically larger than $100 \mu \mathrm{m}$ ) display a rich and complex phenomenology under applied vibrations that has been widely reported. When loosely packed beads are tapped or vibrated the system relaxes slowly eliminating low-density metastable configurations toward a more compact state [1]. Besides this, a large variety of flow and surface instabilities, such as segregation, dilation, bubbling, heaping, flow patterns, surface waves, and arching [2-13] have been observed in vibrated or tapped layers of grains. Particularly interesting for us are the free surface excitations that have been often reported to appear as a result of the momentum transfer associated with intergrain multiple collisions [2-4]. The nucleation of fascinating localized and stable excitations ("oscillons") have been observed in vibrated deep layers of beads [9]. Some of these instabilities are rooted on the action of interstitial gas. The slip force exerted by the surrounding gas on the powder particles scales as $F_{s} \sim 1 / a^{2}$ [14]. In fine powders this force can overcome the particle weight and therefore the interaction with the interstitial gas cannot be neglected. Powder-air interaction has been proved to play a crucial role in the formation of corrugated patterns in a tapped layers of fine powders of particle size $\sim 10 \mu \mathrm{m}$ [13]. Pak et al. [6] also demonstrated that the ambient gas is determinant in generating heaps in vibrated deep beds of glass spheres of size $\leqslant 1 \mathrm{~mm}$. The effect was attributed to the creation of a gap between the grains and the base during the free-flight part of the cycle. Later in the vibration cycle the trapped gas in this gap is compressed providing an upward force on the grains. The application of a vertical gas flow was reported to lift the hysteresis in convective instabilities of horizontally vibrated granular materi- als [10]. When a bed of sand grains $(0.2 \mathrm{~mm}$ in diameter) was shaken at quite large amplitudes bubbling of air pockets, created near the bottom of the bed, was observed by Pak and Behringer [5]. The important effect of surrounding air in the bubble formation was corroborated when bubbles disappeared if the gas was evacuated from the sandbox. Pak and Behringer stressed the analogy of this phenomenon with bubble formation in gas-fluidized beds [15].

If the gas velocity through a stable gas-fluidized bed is further increased above a critical value the two-phase flow becomes unstable and waves of compact regions of low particle concentration (gas bubbles) move upwards through the bed. Hydrodynamic models recognize particle diffusion as the responsible for the stabilization of the fluidized bed since it tends to suppress the amplitude growth of perturbations on local concentration [16]. Bubbling instability has been attributed to the delay in the response of the particles to a change in the local concentration of solids due to the larger particle to gas phase inertia [16]. From the basis of a pure hydrodynamic interaction between fluid and particles, the particle properties involved in the criteria for transition to instability are particle size and density $[16,17]$.

Our work will focus on the effect of vibrations on stable gas-fluidized beds of fine cohesive particles (particle size $\sim 10 \mu \mathrm{m}$ ). These powders can be uniformly fluidized in a very wide interval of solid volume fractions thanks to the use of flow additives that reduce cohesivity. By means of a controlled gas flow we are able to have a precise control on the average solid volume fraction $\phi_{0}$, allowing us to explore a rich phenomenology in the behavior of the vibrated gasfluidized bed as a function of its bulk density. We have shown elsewhere [18] that two clearly differentiated regimes exist in the uniform fluidization interval. For solid volume fractions larger than a certain critical value $\phi_{c}$ the fluidized bed has a mechanical strength. In this solidlike regime particles are static, sustained by enduring contacts, and the pow- 


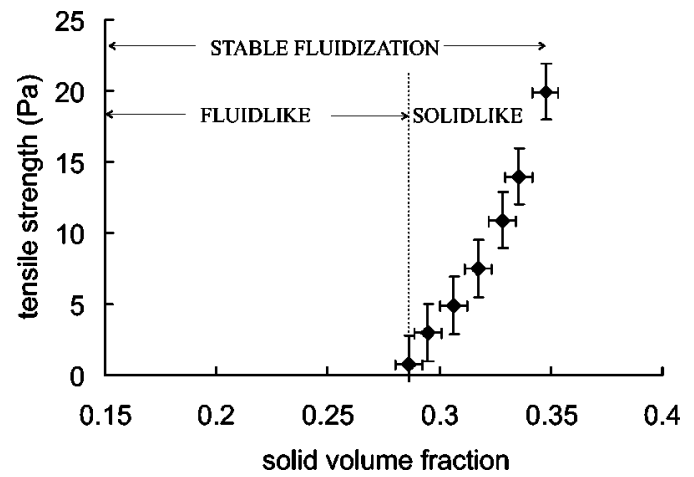

FIG. 1. Tensile strength of the fluidized powder (Canon CLC700 toner) as a function of the solid volume fraction. Data taken from [18].

der has a behavior quite similar to that of a weak solid. If the bed is tilted its free surface remains stable until it avalanches when a critical angle is reached. For $\phi_{0}<\phi_{c}$ particles are no longer in permanent contact with each other. They aggregate due to the domain of interparticle adhesion force over particle weight and aggregates are suspended in the fluidizing gas displaying a diffusive dynamics [18]. The powder in this fluidlike regime takes on many of the properties of a low viscosity fluid, its upper surface remaining horizontal when the container is tilted. Vibration experiments reported in this paper confirm the existence of both regimes since the response of the fluidized bed to vibration depends essentially on which regime the system is. When the bed is in the solidlike regime compaction is observed. On the other hand, when it is in the fluidlike regime we observe an initial expansion followed by either surface or bubbling instability (depending on the value of $\phi_{0}$ ). The critical vibration amplitude for the onset of instability has been measured as a function of the vibration frequency and solid volume fraction. The role of contact forces on the onset of instability will be addressed. Finally we will show that the transition to bubbling can be characterized as a Hopf bifurcation of the stable expanded state.

\section{MATERIALS}

Measurements have been made on a commercially available powder (xerographic toner Canon CLC700). Particle density $\left(\rho_{p}=1199 \mathrm{~kg} / \mathrm{m}^{3}\right)$ was measured by using an AccuPyc 1330 Pycnometer. Toner particles are based on a random copolymer and their volume average diameter is $d_{p}$ $=8.53 \pm 2.32 \mu \mathrm{m}$. They are irregular in shape since they are formed by a grinding process. For the mass of toner used in the experiments the solid volume fraction of the settled powder is $\phi_{0}=\phi_{s} \simeq 0.35$. In the absence of vibration, as the gas flow through the bed is increased and the bed expands, instability in the form of visible bubbling is observed for $\phi_{0}$ $=\phi_{b} \simeq 0.15$. This is the minimum value of $\phi$ that the twophase system can sustain. According to our measurements $[18,19]$ the tensile strength of the fluidized bed becomes negligible for this powder for $\phi_{0}<\phi_{c} \simeq 0.28$ (see Fig. 1). Therefore for $\phi_{0} \in(0.15,0.28)$ the fluidized powder is in the flu-

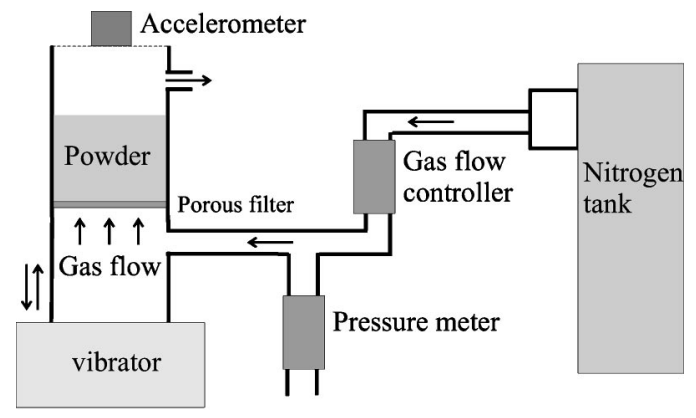

FIG. 2. Sketch of the apparatus.

idlike regime, while for $\phi_{0} \in(0.28,0.35)$ it is in the solidlike regime.

To check for the effect of cohesivity two other xerographic toners (supplied by Xerox Co.) of similar particle size and density as the Canon toner were used. These toners are identical in particle size $(12.7 \mu \mathrm{m}$ diam $)$, particle density $\left(\rho_{p}=1065 \mathrm{~kg} / \mathrm{m}^{3}\right)$, particle resin type (styrenebutadiene), and pigment concentration, but with concentrations of $0.2 \%$ and $0.4 \%$ by weight of surface additive, respectively. This additive (Aerosil R812) consists of agglomerates of nanoparticles of fumed silica. The effect of Aerosil on the interparticle contact forces is described in our previous works [19-21]. The addition of these nanoparticles results in a reduction in the interparticle adhesion force because the additives increase the hardness of the contacts and also reduce the area of contact between grains. In spite of their different cohesivity and in agreement with the predictions of the hydrodynamic models the three toners investigated (of similar particle size and density) show the transition to bubbling instability at approximately the same value of the solid volume fraction $\left(\phi_{b} \simeq 0.15\right)$.

\section{EXPERIMENTAL RESULTS}

\section{A. Experimental setup and procedure}

In Fig. 2 a schematic view of the experimental setup is depicted. The powder sample is held in a 2.52 -cm-diameter cylinder made of polycarbonate, the base of which is a sintered metal filter of $5-\mu \mathrm{m}$ pore size. Dry nitrogen is supplied from a tank of compressed gas and a gas flow controller is used to adjust the flow. By using dry nitrogen we minimize the effect of moisture on the interparticle cohesion. Moreover charge spectrograph measurements made at Xerox Co. indicate that electrostatic forces are minimal. Therefore van der Waals forces are dominant between our dry fine particles. All the measurements start by driving the powder into the freely bubbling phase. Once the powder is in the bubbling phase the fluidizing gas is set down to a given value below the minimum bubbling flow and the powder settles to a reproducible and stable fluidized state. In this way the solid volume fraction of the system was conveniently varied by changing the gas flow rate. The pressure drop across the bed is measured by a differential pressure transducer. The bed height, which was of the order of centimeters (very large compared to particle size), was visualized by a video camera either with a direct or a strobe light. From the height mea- 


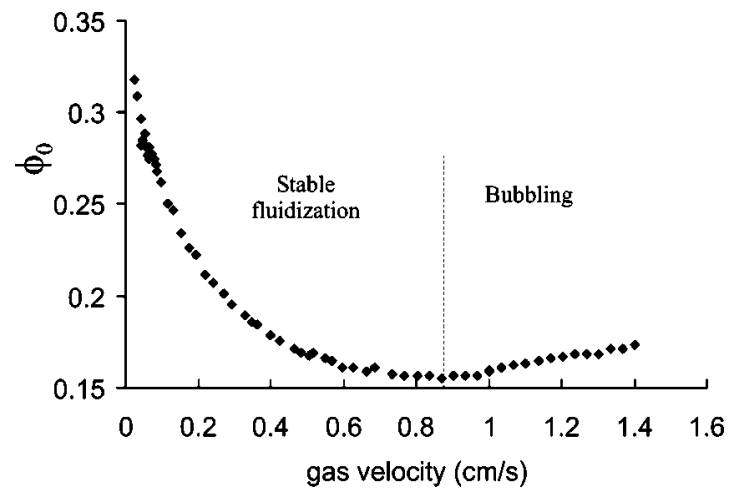

FIG. 3. Solid volume fraction of the fluidized bed (Canon CLC700 toner) as a function of the superficial gas velocity.

sured $h$ and the particle density $\rho_{p}$ we computed the average solid volume fraction, given by $\phi_{0}=m /\left(\rho_{p} S H\right)$, where $m$ $\simeq 6.5 \mathrm{~g}$ is the mass of the powder sample and $S$ is the area of the filter. In Fig. $3 \phi_{0}$ is represented as a function of the superficial gas velocity $v_{g}$. For $v_{g}>0.8 \mathrm{~cm} / \mathrm{s}$ (minimum gas velocity for bubbling) the mean bed height begins to decrease slowly (i.e., $\phi_{0}$ increases), with marked oscillations of the free surface as gas bubbles burst.

The powder bed was mounted on an electromagnetic vibration exciter driven by a signal generator, which provided sinusoidal, vertical vibrations of controlled frequency $f$, and amplitude $A$. The frequency of the oscillations could be controlled to within $\pm 0.1 \mathrm{~Hz}$ in the range from 10 to $1000 \mathrm{~Hz}$. The amplitude of vibrations is monitored using a piezoelectric accelerometer glued on the top of the vessel. Throughout the experiments reported here the powder was fluidized to a given value of the solid volume fraction $\phi_{0}$ by a given gas flow and then the amplitude of vibration was slowly increased from zero at a fixed frequency.

\section{B. The solidlike regime}

When the powder is fluidized at superficial gas velocities below $0.1 \mathrm{~cm} / \mathrm{s}$ the solid volume fraction is above 0.28 , permanent contacts form, and the bed behaves as a weak solid. If vibration is applied in this regime the bed relaxes slowly towards a more compact solid structure in spite of the presence of a gas flow whose superficial velocity is above the minimum fluidization gas velocity (which is about $\sim 0.01 \mathrm{~cm} / \mathrm{s}$ ). The compaction process is irreversible, i.e., if vibration is stopped the bed does not return to its original density. The behavior of the powder bed in this regime is quite similar to the compaction observed when loose packed beads are tapped or vibrated [1].

\section{The fluidlike regime}

In this regime permanent contacts do not exist anymore, particle aggregates are freely suspended in the gas and stresses are carried by the interstitial fluid and by collisions. We observe that when vibration is applied to the bed in this regime the bed expands, i.e., the solid volume fraction decreases. Fine particles are not freely floating as individuals in the fluidlike fluidized bed but are agglomerated due to attrac-

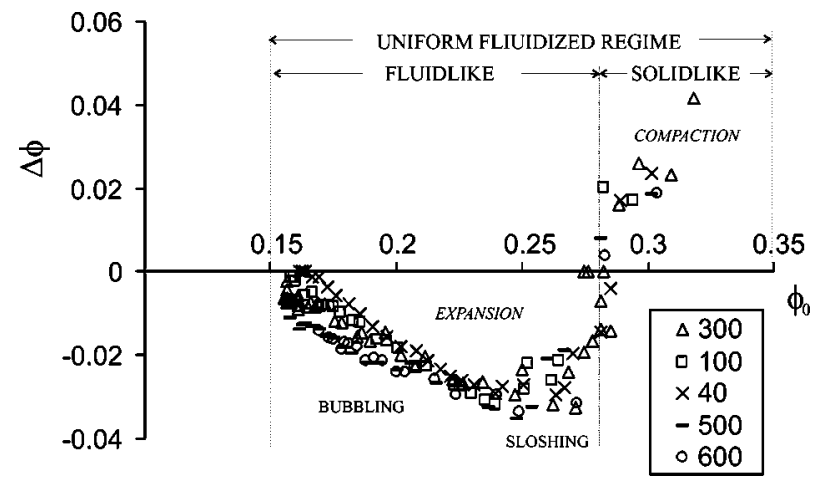

FIG. 4. Variation of the solid volume fraction when the uniform fluidized bed is vibrated from the initial state characterized by a solid volume fraction $\phi_{0}$. Each series of data corresponds to a vibration frequency indicated in the inset in $\mathrm{Hz}$. The data in the fluidlike regime corresponds to the maximum decrease in the solid volume fraction (just before the onset of instability, either sloshing or bubbling). The data in the solidlike regime indicates the increase of the solid volume fraction due to compaction of the fluidized bed.

tive van der Waals force [22,23]. On the other hand, it has been shown that, for highly cohesive powders, vibration of the bed improves the fluidization quality by helping the gas flow to overcome cohesive forces and break up channels that conduct fluidizing gas away from the bulk and prevent fluidization [24]. In our case of homogeneously fluidized aggregated particles the expected effect of vibration would be to increase the rate of collisions between aggregates, promoting their disruption. As a result there would be an improvement of the gas-solid contact, the slip force (which is inversely proportional to the square of the aggregate radius) on the reduced aggregates would be larger, and consequently the stable bed would expand.

However, the expansion stops when a critical vibration amplitude $A_{c}$, which depends on the vibration frequency, is reached. Then two kinds of instabilities, depending on the value of the solid volume fraction, have been observed. In Fig. 4 we have plotted the change in the solid volume fraction $\Delta \phi=\phi_{m}-\phi_{0}$ until the bed destabilizes (or compaction stops if it is in the solidlike regime) as a function of the initial solid volume fraction $\phi_{0}$ for a set of runs made at different frequencies $f$. This figure shows that $\Delta \phi$ depends essentially on the initial value of $\phi_{0}$ and is almost independent on $f$, except for states of $\phi$ close to 0.15 (for which bubbling occurs spontaneously in the absence of vibrations) whose expansion is slightly higher at larger frequencies. Moreover the boundary between the solidlike $(\Delta \phi>0)$ and the fluidlike $(\Delta \phi<0)$ regimes is clearly seen at $\phi_{0} \simeq 0.28$. As we should expect, when $\phi_{0}$ approaches $0.15, \Delta \phi \rightarrow 0$. Thus $\Delta \phi$ is zero for $\phi=0.15$ and $\phi=0.28$ (boundaries of the fluidlike regime). Therefore $\Delta \phi$ must have a minimum in between and we find it at $\phi_{0} \simeq 0.24$, independently of the vibration frequency. For this value of the initial solid volume fraction the maximum expansion of the bed occurs. In Fig. 5 the initial $h_{0}$ and maximum $h_{m}$ bed heights are plotted as a function of $\phi_{0}$. This figure shows that $h_{m}$ can be well approximated by a linear function of $\phi_{0}$. Taking into account that $h=m /\left(\rho_{p} S \phi\right)$ we derive for the minimum solid volume 


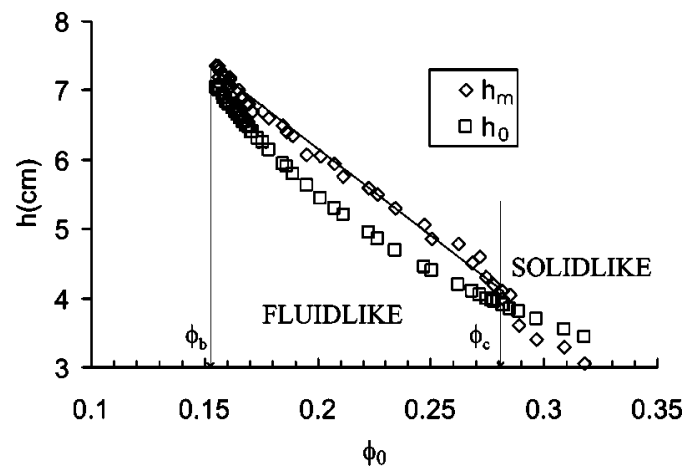

FIG. 5. Initial bed height $h_{0}$ of the fluidized bed and maximum height $h_{m}$ of the expanded bed when vibration is applied as a function of the initial solid volume fraction $\phi_{0}$. At $\phi_{0}=\phi_{c}$ the fluidized bed enters the solidlike regime. At $\phi_{0}=\phi_{b}$ the nonvibrated bed enters the bubbling regime if the gas flow is further increased. Only data for $f=300 \mathrm{~Hz}$ has been represented for the sake of clarity.

fraction $\phi_{m}$ of the expanded bed the simple expression

$$
\frac{1}{\phi_{m}} \simeq \frac{1}{\phi_{c}}\left(1+\frac{\phi_{c}-\phi_{0}}{\phi_{b}}\right)
$$

where $\phi_{c} \simeq 0.28$ is the critical solid volume fraction for the transition to the solidlike regime and $\phi_{b} \simeq 0.15$ is solid volume fraction for the transition to bubbling in the absence of vibration.

\section{Free surface instability}

In the interval $\phi_{0} \in(0.24,0.28)$, close to the solidlike regime, free surface instability (sloshing) is observed when the vibration amplitude reaches a critical value $A_{c}$. The evolution of the system around the critical vibration amplitude is completely reversible, i e. no hysteresis have been found. This instability becomes more apparent at low frequencies $(f<100 \mathrm{~Hz})$ for which $A_{c}$, as we will see below, is larger. Furthermore at low frequencies we observe the nucleation of localized sloshing that reminds of solitary waves ("oscillons") observed in vibrated deep beds of noncohesive beads [9]. Likewise sloshing is reminiscent of the surface instabilities observed in vibrated [3] or tapped [13] fine powders. Surface waves induced by vibration were reported to be specially visible at very large vibration amplitudes [3]. Duran [13] observed that fine particles on the free surface were expelled at every tap and attributed it to the effect of the upward flow of the trapped air in the powder. Moreover he reports on some interesting complex patterns, like wrinkled volcanos or herringbone-shaped structures, when a continuous air flow was passed through the tapped powder layer.

\section{Bubbling instability}

One of the effects of increasing the gas flow in the fluidlike regime is the decrease of the average solid volume fraction. The other is the increase of the granular temperature $T$ $[18,25]$, which is defined in terms of the ensemble average of the squared fluctuation velocity, $3 T=\left\langle\mathbf{v}^{2}\right\rangle-\langle\mathbf{v}\rangle^{2}[18]$. The granular temperature can be estimated by means of the ef-

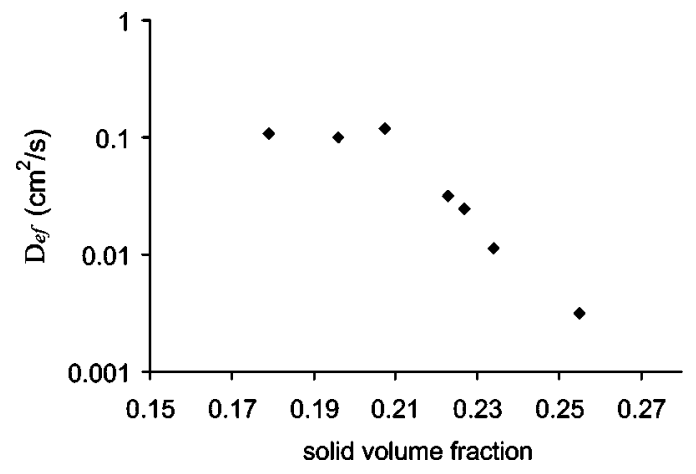

FIG. 6. Effective diffusion coefficient $D_{e f}$ of the fluidized bed in the fluidlike regime $[\phi \in(0.15,0.28)]$ as a function of the solid volume fraction (data taken from [18]). In the solidlike regime particles are static and the diffusion coefficient is zero.

fective diffusion coefficient, $D_{e f}=l \sqrt{T}$, where $l$ represents the mean free path. We have presented elsewhere [18] measurements of $D_{e f}$ of the toner Canon CLC700 in the fluidlike regime. Values for $D_{e f}$ were obtained from measuring the mixing rate of two fluidized samples of different color. The results are shown in Fig. 6. According to Fig. 6 particle velocity fluctuations are enhanced by the increase in the gas flow until $\phi$ is around 0.21 . Particle diffusivity then reaches a plateau in spite of the increase of gas flow (and the consequent reduction in $\phi$ ). We expect that the collisions rate should be favored by the increase in velocity fluctuations. At $\phi_{0}=0.24$ when vibration is applied the bed expansion is maximum and $\phi$ reaches a minimum value of $\phi \simeq 0.21$ (see Fig. 4). Interestingly this value coincides with the value of $\phi$ for which we measure a saturation in diffusivity (see Fig. 6). The proponents of hydrodynamic models argue that particle diffusion plays a decisive role in stabilizing the fluidized bed against local perturbations on the concentration of solids [16]. According to this, as the gas flow is further increased and $\phi_{0}$ is reduced below 0.24 the saturation in particle diffusivity would reduce the ability of the system to suppress disturbances in the local concentration of particles. In other words, lowering $\phi$ below 0.21 accelerates the approach of the flow of the system to the critical state. From this plausible argument it can be understood that for $\phi<0.21$ the perturbation of the system caused by vibration promote flow instability and that the maximum expansion of the bed decreases as $\phi$ is further reduced. Accordingly, when $\phi_{0}$ is reduced below 0.24 , surface sloshing is not the first instability observed. Instead the two-phase flow becomes unstable and visible bubbling appears. Figure 7 displays the evolution of the solid volume fraction in a typical experiment in which bubbling instability is observed. When the vibration amplitude approaches a critical value $A_{c}$ the system reaches its maximum expansion and $\phi$ its minimum value $\phi_{m}$. Then part of the gas is trapped from the dense phase by bubbles and $\phi$ increases, with oscillations of the free surface due to gas bubbles burst. The transition to instability is not sharp. There is an indeterminacy (see bar plotted in Fig. 7) in the measurement of $A_{c}$ due to our sensitivity to detect an increase of $\phi$ (the error bar of the measured values of $\phi$ are shown in Fig. 7). Hereafter we take for $A_{c}$ the value of the 


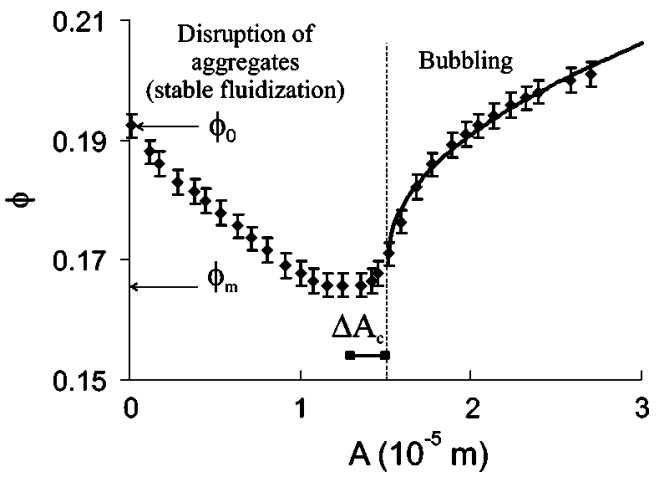

FIG. 7. Evolution of the solid volume fraction $(\phi)$ of the fluidized bed as the vibration amplitude $(A)$ is increased for a typical experiment (gas velocity $0.334 \mathrm{~cm} / \mathrm{s}$, vibration frequency $200 \mathrm{~Hz}$ ). The solid line is a square root fit. The bar size indicates the typical indeterminacy $\left(\Delta A_{c}\right)$ on $A_{c}$.

right end of the bar in Fig. 7 since at that value the increase in $\phi$ is more marked and is easily detected. As the vibration amplitude is further increased above $A_{c}, \phi$ keeps growing and the oscillations of the free surface become more noticeable due to the increase of bubbles size. We have observed a correlation between the typical size of the free surface oscillations and the imposed frequency. As the vibration frequency is increased the oscillations become less marked going from several millimeters at low frequencies $(f$ $\lesssim 100 \mathrm{~Hz}$ ) to less than $1 \mathrm{~mm}$ at high frequencies ( $f$ $\gtrsim 500 \mathrm{~Hz}$ ). Parallel to the bubbling instability we observe the onset of a modest convective motion on the free surface that can be related to the reported convection of the top layers of shaken fine powders $[10,11]$, to the circulation of solids generated by bubbling [14], or maybe to both. Indeed the evolution of the system for $A>A_{c}$ resembles its evolution in the absence of vibration when the gas flow is increased above the minimum bubbling flow (see Fig. 3).

The transition to bubbling instability does not show any hysteresis on cycling through the onset of bubbling. The average solid volume fraction is a continuous reversible function of the vibration amplitude. Tennakoon et al. [10] reported also on the absence of hysteresis in convective instabilities of horizontally vibrated granular beds when a gas flow through the system was applied. Thus the suppression of hysteresis by the use of a gas flow seems to be a common factor independent of the dynamic instability involved. Tennakoon et al. conjectured that the reduction of the static friction was the reason for removing hysteresis at the transition to convective instability [10]. In our experiment the powder in the fluidlike regime has no static friction and according to the same argument we should not expect any hysteresis. The scenario is quite different when the fluidized powder is in the solidlike regime where the powder retains a certain mechanical strength. In such a static regime the compaction observed when the bed is vibrated is irreversible.

The formation of gas bubbles in vibrated beds of sand grains without an applied gas flow was already observed by Pak and Behringer [5]. An interesting feature of that work, analogous to ours in gas-fluidized beds, is the reported in-

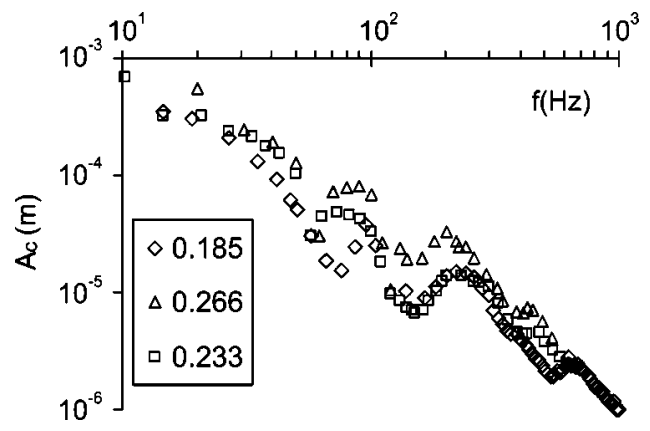

FIG. 8. Dependence of the critical vibration amplitude $A_{c}$ for the onset of instability on the vibration frequency $f$. Each series corresponds to an initial solid volume fraction indicated in the inset.

crease of bubble size as the vibration amplitude is further increased. It must be remarked however that, as Pak and Behringer report [5], the formation of bubbles in the absence of gas flow needs for exaggerated vibration amplitudes, and prior to bubbling, convection and surface instabilities (sloshing) take place. Actually we have reproduced this experiment in our laboratory by using glass beads (average size $50 \mu \mathrm{m}$ ) and pronounced surface convection, heaping, and sloshing were observed before the appearance of bubbles. However, when we tried to repeat the experiment with cohesive powder, the initial compaction induced by vibration made the powder so cohesive that we were not able, with our instrumentation, to achieve strong enough shaking to break interparticle contacts.

\section{Critical vibration amplitude}

In this section we will discuss the dependence of the critical amplitude for the onset of instability (either surface or flow instability) $A_{c}$ on the vibration frequency $f$ and on the solid volume fraction $\phi_{0}$. In Fig. 8 we have plotted $A_{c}$ as a function of $f$ for three states of different initial concentration of solids. $A_{c}$ is well fitted by a potential law $A_{c}$ $\simeq \gamma f^{-1.3}, \quad \gamma$ being a weakly dependent function of $\phi$. However $A_{c}$ presents some puzzling peaks at certain special frequencies $f_{p}(100,200,400$, and $600 \mathrm{~Hz})$. Interestingly, these peaks do not depend essentially on the solid volume fraction (see Fig. 8), nor on the powder cohesivity (see Fig. 9).

To have further insight on the dependence of $A_{c}$ on $\phi_{0}$ we present in Fig. 10 the critical vibration amplitude as a function of the solid volume fraction for a set of runs performed at different fixed frequencies. As could be anticipated from Fig. 8, Fig. 10 does not show a strong dependence of $A_{c}$ on $\phi_{0} . A_{c}$ changes smoothly as the solid volume fraction is decreased. When $\phi_{0}$ reaches 0.15 the bed bubbles in the absence of vibration and $A_{c}$ falls sharply to zero. At low frequencies $A_{c}$ decreases as $\phi_{0}$ is decreased. However, this tendency changes at high frequencies for which $A_{c}$ is almost independent of $\phi_{0}$.

Measurements of the bubble frequency $N_{b}$ (defined as the number of bubbles that rise up per unit cross section of the bed per unit time) of freely bubbling nonvibrated fluidized beds have been reported in the literature [26] and the typical 


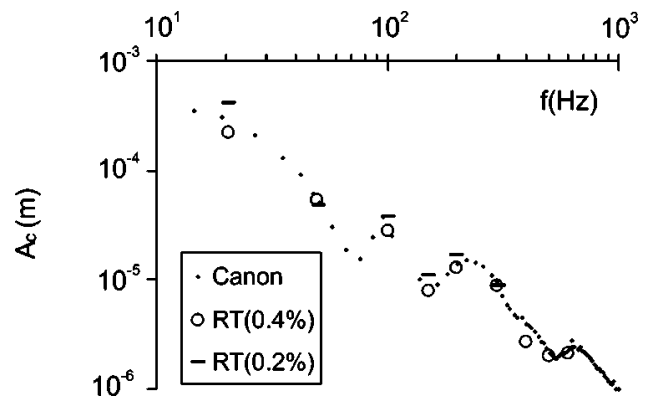

FIG. 9. Critical vibration amplitude $A_{c}$ for the onset of instability as a function of the vibration frequency $f$ for three powders of similar particle size and density but different particle surface structure. RT toners are model toners characterized by the weight $\%$ of surface additive. The initial solid volume fraction for the three powders tested is $\phi_{0}=0.185$.

values are below $10 \mathrm{~Hz}$. Presumably the typical frequencies of the larger perturbations in the local solids concentration in the stable fluidized bed are of the same order of magnitude. The magnitude of these perturbations are expected to increase as the solid volume fraction decreases until they become finite and the bed bubbles. The natural frequency of the perturbations is $f_{n} \sim c / \lambda, \quad c$ being the perturbation characteristic wave speed and $\lambda$ its wavelength. Thus if the stable fluidized bed is vibrated at low frequencies the growth of the largest perturbations is expected to be promoted. Accordingly we should expect a decrease of the critical amplitude for bubbling as the solid volume fraction is reduced, i.e., as local perturbations increase in magnitude. Moreover we should expect, as we observe, large fluctuations on the free surface when bubbling is promoted by vibration. On the other hand, if the bed is vibrated at very high frequencies, quite larger than the typical natural frequency of bubbles, the perturbations magnified for the onset of bubbling would be of large frequency, small size, and presumably weakly dependent on the average solid volume fraction. This would explain the observed independence of $A_{c}$ on $\phi_{0}$ and the very small amplitude of the oscillations (tenths of a millimeter) of the free surface at large vibration frequencies. Note also that, for $f>500 \mathrm{~Hz}, \quad A_{c}$ is smaller than the size of the particles, which in turn is of the order of the average interparticle separation in the fluidized state. Thus, at large frequencies,

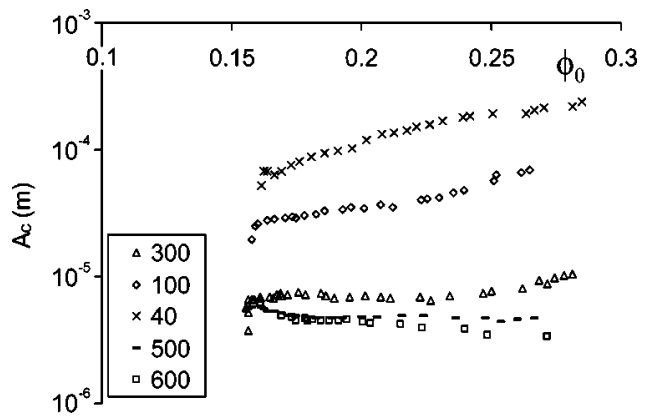

FIG. 10. Critical vibration amplitude $A_{c}$ for the onset of instability as a function of the solid volume fraction $\phi_{0}$. Each series corresponds to a fixed vibration frequency indicated in the inset in $\mathrm{Hz}$. vibrations cannot be transmitted by interparticle contacts and this provides another explanation for the independence of $A_{c}$ on the average solids concentration.

\section{The role of contact forces}

From a theoretical point of view the stabilization mechanism for uniform fluidization was long ago attributed to an effective particulate-phase pressure in the momentum equation that tends to resist variations in the local solid volume fraction and thereby has a stabilizing influence [27]. Yet, the physical origin of the particle-phase pressure has been a subject of strong debate until very recently [28,29]. Rietema [30] outlined that interparticle contact forces give an effective elastic modulus to the bed that stabilize the system against small disturbances. Tsinontides and Jackson [15] joined Rietema's arguments and stated that the loss of strength of the assembly was responsible for the loss of stability and the consequent onset of bubbling. However this argument would imply that the particle assembly should have a yield stress through all the interval of stability and should not behave in a fluidlike way but rather like a weak solid. It also implies that the transition to instability would be very sensitive to factors that affect contact forces, such as the surface properties in dry fine powders, for which cohesion between particles arise mainly from dispersion or van der Walls forces. Strongly opposed to Rietema's ideas, Foscolo and Gibilaro [17] proposed a stability mechanism of hydrodynamic origin. An alternative stabilizing mechanism rooted on hydrodynamic arguments was suggested by Batchelor [16]. The physical origin of this mechanism is based on the existence of random fluctuations in the particles velocities that are linked to the diffusion of particles [16,29]. According to hydrodynamic models the only particle properties that affect essentially the instability criteria are their size and density.

In our previous work [18] we have shown that the fluidized powder behaves like a fluid (it does not exhibit any yield stress) and displays a diffusive dynamics in a broad interval of uniform fluidization previous to the onset of instability. Thus, according to that work, the source of instability cannot be attributed to the loss of mechanical strength but rather to hydrodynamic interactions. Here we find another evidence to support the hydrodynamic origin of bubbling instability. Even though the source of bubbling in our system is not the increase of gas flow beyond a certain limit but the excitation of the system by external vibration, we should expect that if contact forces were relevant in the transition to instability we would find a marked dependence of the critical vibration amplitude for bubbling on the powder cohesivity. In Fig. 9 results obtained for $A_{c}$ on Canon CLC700 toner are compared with some results obtained on other powders of similar particle size and density but with different cohesivity. These toners are blended with different amounts of surface additive such as Aerosil ${ }^{\circledR}$ (nanoparticles of fumed silica), well known for its ability to control interparticle cohesive forces [19]. As can be seen from Fig. 9 the values obtained for the critical vibration amplitude are on the same curve for the three powders investigated. This result is in opposition to the proposed stabilizing mechanism based on contact forces 

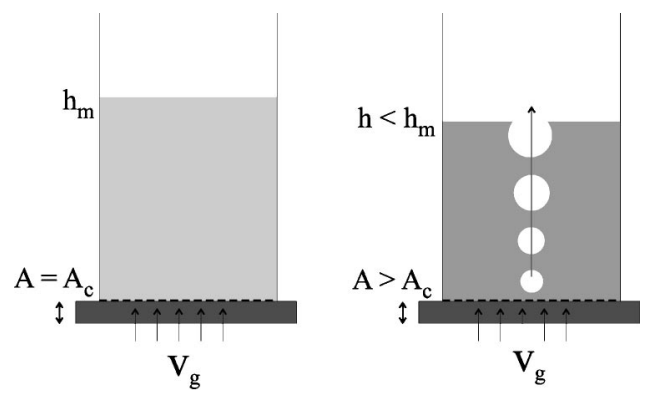

FIG. 11. Schematic picture of the nucleation of bubbles in vibrated gas-fluidized beds. When the vibration amplitude $A$ reaches the critical value $A_{c}$ part of the gas rises in bubbles. When the bubbles burst on the free surface it collapses and oscillates according to the bubble frequency.

$[15,30]$. On the contrary they support the idea that the stabilizing mechanism must have a hydrodynamic origin $[16,17]$.

\section{E. The transition to bubbling as a Hopf bifurcation}

In a typical experiment, when the fluidlike fluidized bed is vibrated, the value of $\phi$ decreases due to disruption of aggregates from the initial value $\phi_{0}$ down to $\phi_{m}$. Then a critical amplitude $A=A_{c}$ is reached for which bubbling instability occurs. As $A$ is further increased the size of the bubbles (as measured by the free surface oscillations) and the average solid volume fraction increase continuously. Pak and Behringer [5] related the bubbling instability observed in a vibrated sandbox in the absence of imposed gas flow with a Hopf bifurcation. On the other hand, the transition to bubbling instability in nonvibrated gas fluidized beds as the gas flow is increased has been described theoretically also as a Hopf bifurcation of the uniform state [31]. Following Pak and Behringer [5] we can consider the bubble size as a measure of the amplitude of the oscillations of the system. The nucleation of bubbles as the gas velocity is kept constant gives, as a result, a densification of the dense phase (see Fig. 11). Part of the gas flow that was carried through the dense phase is now captured by the gas phase (bubbles). Gas flows up through the bed and shortcuts between the bubbles with a high velocity. When the gas bubbles burst on the free surface the bed height $h$ decreases and oscillates. The rising velocity of bubbles has been correlated to the bubble size $R$ by $U_{b}$ $\propto \sqrt{R}$ [32]. Thus as bubbles grow in size and their velocity increases the height decrement $h-h_{m}$ and the size of oscillations would also increase. The total mass of solids in the bed must be constant, hence $\phi_{m} h_{m}=\phi h$. From this balance we get $\left|h-h_{m}\right| / h_{m}=\left|\phi_{m}-\phi\right| / \phi \equiv \Delta \phi_{b} / \phi$. Therefore the increment of the average solid volume fraction $\Delta \phi_{b}$ can be taken as a measure of the size of bubbles that can be in turn considered a measure of the amplitude of the oscillations of the system. From Fig. 7 it can be seen that once the instability is reached the variation of the solid volume fraction can be very well fitted by the square root law $\Delta \phi_{b}=\alpha(A$ $\left.-A_{c}\right)^{1 / 2}$. In Fig. 12, $\Delta \phi_{b}$ is plotted against $\left(A-A_{c}\right)^{1 / 2}$ for a set of experiments made at different values of $\phi_{0}$ and at different frequencies. In all the runs the experimental data
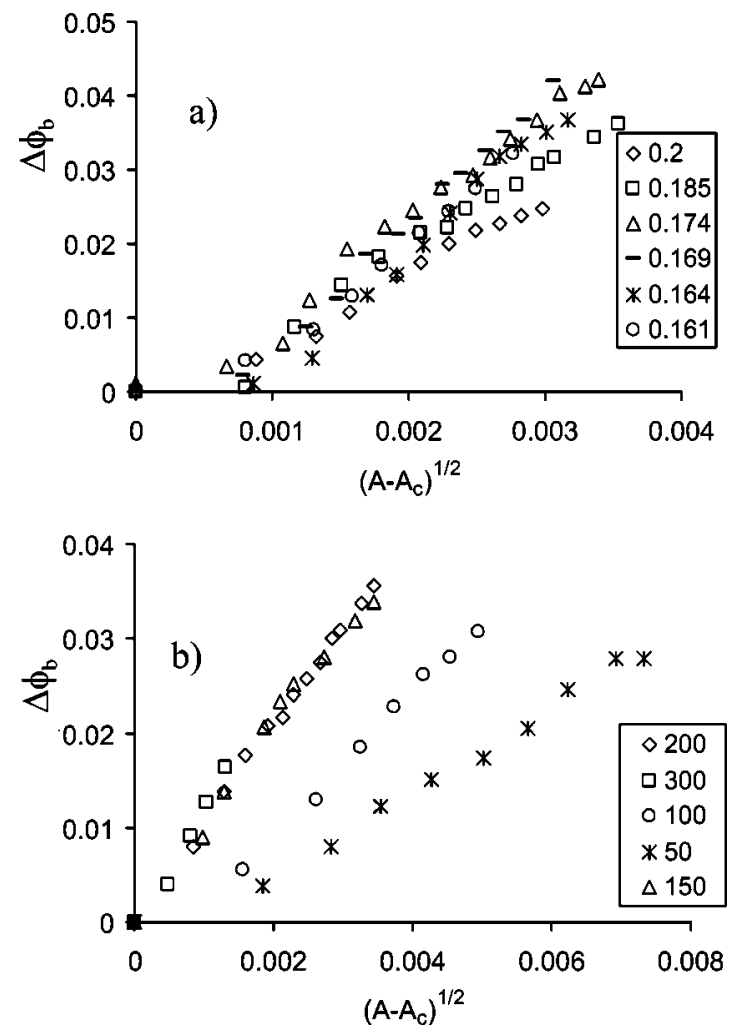

FIG. 12. Variation of the average solid volume fraction $\left(\Delta \phi_{b}\right.$ $=\phi-\phi_{m}$, see Fig. 7) after bubbling instability is reached as a function of the square root of the bifurcation parameter $\left(A-A_{c}\right)$. (a) States of different initial solid volume fraction $\phi_{0}$ (indicated in the figure) vibrated at the same frequency $f=200 \mathrm{~Hz}$. (b) States of the same initial solid volume fraction $\phi_{0}=0.18$ vibrated at different frequencies (indicated in the figure in $\mathrm{Hz}$ ).

are pretty well fitted by a linear law, suggesting that bubbling instability in vibrated gas-fluidized beds can also be related to a Hopf bifurcation.

\section{CONCLUSIONS}

We have investigated the behavior of uniformly fluidized fine cohesive powders (particle size $\sim 10 \mu \mathrm{m}$ ) subjected to vibrations. These powders show a wide interval of stable fluidization [solid volume fraction $\left.\phi_{0} \in(0.15,0.35)\right]$ as opposed to coarse grains for which bubbling appears soon after the minimum gas velocity for fluidization is reached. In the interval of uniform fluidization the powder exhibits two regimes. In the solidlike regime $\left(0.28<\phi_{0}<0.35\right)$ particle contacts are permanent and the expanded fluidized bed has a mechanical strength. When the bed in this regime is vibrated it compacts much in the same way as loose static beds of noncohesive grains do. In the fluidlike regime $\left(0.15<\phi_{0}\right.$ $<0.28)$ there are not enduring contacts and aggregates of particles are freely suspended in the fluid. When the bed in this regime is vibrated it initially expands due to aggregates disruption induced by vibration. However a critical vibration amplitude $A=A_{c}$ is reached at which the bed destabilizes. Two kinds of instabilities have been observed. Sloshing of the free surface occurs for $\phi_{0} \in(0.24,0.28)$. In the interval 
$\phi_{0} \in(0.15,0.24)$ the two-phase flow becomes unstable and part of the gas rises in bubbles. Then the free surface oscillates and the average solid volume fraction increases. $A_{c}$ scales as $f^{-1.3}$ and depends weakly on $\phi$. The amplitude of the oscillations of the free surface, which are a measure of the size of the gas bubbles, decrease as the vibration frequency $f$ is increased, going from several millimeters at low $f(f<100 \mathrm{~Hz})$ to tenths of millimeter at large $f(f$ $>500 \mathrm{~Hz}$ ). If $A$ is further increased above $A_{c}$ the decrease in the average solid volume fraction scales as $\left(A-A_{c}\right)^{1 / 2}$. Thus the transition to bubbling instability resembles a Hopf bifurcation of the stable state. We have checked that $A_{c}$ does not depend on the powder cohesivity as particle size and density are held constant. This finding can be taken as a further evidence for the invalidity of stability criteria proposed in the literature that are based on the existence of a mechanical strength in the fluidized bed.

\section{ACKNOWLEDGMENTS}

We thank Dr. A. T. Pérez for fruitful discussions. This research was supported by the Xerox Foundation, Spanish Government Agency Dirección General de Ciencia y Tecnologí a (DGES) under Contract No. BMF2000-1056 and NATO Grant No. LINKAGE PST.CLG.976575.
[1] E. R. Nowak, J. B. Knight, E. Ben-Naim, H. M. Jaeger, and S. R. Nagel, Phys. Rev. E 57, 1971 (1998); C. Josserand, A. V. Tkachenko, D. M. Mueth, and H. M. Jaeger, Phys. Rev. Lett. 85, 3632 (2000).

[2] S. Douady, S. Fauve, and C. Laroche, Europhys. Lett. 8, 621 (1989).

[3] H. Pak and R. P. Behringer, Phys. Rev. Lett. 71, 1832 (1993).

[4] F. Melo, P. Umbanhowar, and H. L. Swinney, Phys. Rev. Lett. 72, 172 (1994).

[5] H. Pak and P. R. Behringer, Nature (London) 371, 231 (1994).

[6] H. Pak, E. Van Doorn, and R. P. Behringer, Phys. Rev. Lett. 74, 4643 (1995).

[7] C. R. Wassgren, C. E. Brennen, and M. L. Hunt, J. Appl. Mech. 63, 712 (1996).

[8] E. van Doorn and R. P. Behringer, Europhys. Lett. 40, 387 (1997).

[9] P. B. Umbanhowar, F. Melo, and H. L. Swinney, Physica A 249, 1 (1998).

[10] S. G. K. Tennakoon, L. Kondic, and R. P. Behringer, Europhys. Lett. 45, 470 (1999).

[11] E. Falcon, K. Kumar, K. Bajaj, and J. K. Bhattacharjee, Phys. Rev. E 59, 5716 (1999).

[12] C. Bizon, M. D. Shattuck, and J. B. Swift, Phys. Rev. E 60, 7210 (1999).

[13] J. Duran, Phys. Rev. Lett. 84, 5126 (2000).

[14] K. Rietema, The Dynamics of Fine Powders (Elsevier, London, 1991).

[15] S. C. Tsinontides and R. Jackson, J. Fluid Mech. 255, 237 (1993).

[16] G. K. Batchelor, J. Fluid Mech. 193, 75 (1988).

[17] P. U. Foscolo and L. G. Gibilaro, Chem. Eng. Sci. 39, 1667 (1984).
[18] J. M. Valverde, A. Castellanos, and M. A. S. Quintanilla, Phys. Rev. Lett. 86, 3020 (2001).

[19] J. M. Valverde, A. Ramos, A. Castellanos, and P. K. Watson, Powder Technol. 97, 237 (1998); P. K. Watson, J. M. Valverde, and A. Castellanos, ibid. 115, 44 (2001); J. M. Valverde, A. Castellanos, and P. K. Watson, ibid. (to be published).

[20] A. Castellanos, J. M. Valverde, A. T. Perez, A. Ramos, and P. K. Watson, Phys. Rev. Lett. 82, 1156 (1999).

[21] J. M. Valverde, A. Castellanos, A. Ramos, and P. K. Watson, Phys. Rev. E 62, 6851 (2000).

[22] T. Zhou and L. Hongzhong, Powder Technol. 101, 57 (1999).

[23] J. M. Valverde, M. A. S. Quintanilla, A. Castellanos, and P. Mills, Europhys. Lett. 54, 329 (2001).

[24] E. Marring, A. C. Hoffman, and L. P. B. M. Janssen, Powder Technol. 79, 1 (1994).

[25] G. D. Cody, D. J. Goldfarb, G. V. Storch, Jr., and A. N. Norris, Powder Technol. 87, 211 (1996).

[26] C. E. J. van Lare, H. W. Piepers, J. N. Schoonderbeek, and D. Thoenes, Chem. Eng. Sci. 52, 829 (1997); J. Wiman and A. E. Almstedt, ibid. 53, 2167 (1998); H. Enwald, E. Peirano, A. E. Almstedt, and B. Leckner, ibid. 54, 311 (1999).

[27] T. B. Anderson and R. Jackson, Ind. Eng. Chem. Fundam. 8, 137 (1968)

[28] N. Menon and D. J. Durian, Phys. Rev. Lett. 79, 3407 (1997).

[29] Y. A. Buyevich and G. D. Cody, in Proceedings of the 3rd World Congress on Particle Technology, Brighton, UK, 1998 (IChemE, Brighton, UK, 1998), produced by Catalyst Electronic Publishing on CD, p. 207.

[30] K. Rietema, Chem. Eng. Sci. 28, 1493 (1973).

[31] B. J. Glasser, I. G. Kevrekidis, and S. Sundaresan, J. Fluid Mech. 306, 183 (1996); B. J. Glasser, S. Sundaresan, and I. G. Kevrekidis, Phys. Rev. Lett. 81, 1849 (1998).

[32] P. N. Rowe and R. Matsuno, Chem. Eng. Sci. 26, 923 (1971). 